\title{
RAZÕES QUE LEVAM ADOLESCENTES GRÁVIDAS A PERMANECEREM NAS CONSULTAS PRÉ-NATAIS NUMA MATERNIDADE PÚBLICA DE SALVADOR
}

\author{
Tamires Guedes Costa da Silva ${ }^{a}$ \\ Thami kristine Timm Antunes ${ }^{b}$ \\ Rita de Cássia Calfa Vieira Gramacho"
}

\begin{abstract}
Resumo
A gravidez na adolescência vem sendo considerada um problema de saúde para a sociedade brasileira, por ocorrer na maioria das vezes de forma extemporânea e não planejada. As dificuldades para enfrentar as modificações relacionadas à gestação em uma fase de incertezas e transformações biopsicossociais podem ser agravantes para a aceitabilidade e comprometimento com a gestação. $\mathrm{O}$ objetivo do estudo foi identificar as razões que levam as adolescentes grávidas a permanecerem nas consultas pré-natais. Utilizado como linha metodológica abordagem qualitativa, quantitativa e exploratória, a amostra foi composta por 15 adolescentes, dentre elas, 10 gestantes e 05 puérperas. Como resultados pôde-se depreender que a maioria das gestantes vive em um contexto social de completa dependência financeira e que muitas sofrem influência direta da família na tomada de decisões. Consideramos que os anseios e cuidados com a saúde do bebê contribuíram no maior índice de resultados que motivaram as adolescentes a frequentarem o pré-natal.
\end{abstract}

Palavras-chave: Gravidez na adolescência; Pré-natal; Enfermagem.

a. Enfermeira graduada pela Universidade Jorge Amado, Enfermeira Assistencial na UTI Pediátrica do Hospital Santo Antonio. Salvador-Ba. (linkaguedes29@bol.com.br)

b. Enfermeira graduada pela Universidade Jorge Amado. (thamitim@hotmail.com)

c. Enfermeira Obstetra e Docente do curso de Enfermagem da Universidade Jorge Amado. Diretora Geral da Maternidade Púbica Tsylla Balbino e Presidente da Abenfo-Ba. (ritacalfa@hotmail.com) Artigo apresentado como Trabalho de Conclusão de Curso da Graduação em Enfermagem. 


\title{
REASONS THAT LEAD PREGNANT TEENAGERS TO STAY ON PRENATAL CONSULTATION IN A PUBIC MATERNITY AT SALVADOR
}

\begin{abstract}
The teenage pregnancy has been considered a health problem to the brazilian society, by occurring mostly in a extemporaneous and not planned form. The difficulties to confront the modifications related to the gestation in a fase of ncertainties and biopsychosocial transformations can be aggravating to the acceptability and ommitment with the gestation. The goal of the study was to identify the reasons that ead the pregnant teenagers to stay in the prenatal consultations. Using as etodological line the qualitative methodological approach, quantitative and exploratory approach, the sample was composed by 15 teenagers, between them 10 pregnants and 05 puerperals. With the results, could be inferred that the majority of the pregnant teenagers lives in a social context of complete finacial dependency and many suffer direct influency of the family in the decision-making. We considered that the yearnings and cares with the baby's health contributed in the biggest index of the results that motivated the teenagers to attend to the prenatal.
\end{abstract}

Keywords: Teenage pregnancy; Prenatal; Nursing.

\section{RAZONES PRINCIPALES ADOLESCENTES EMBARAZADAS PERMANECEN EN UNA CONSULTA PRENATAL MATERNIDAD EN PÚBLICO SALVADOR}

\begin{abstract}
Resumen
Embarazo en la adolescencia se ha considerado un problema de salud para la sociedad brasileña, se producen más a menudo en forma extemporánea y no planificado. Las dificultades para hacer frente a los cambios relacionados con el embarazo en un período de incertidumbre y cambios biopsicosociales pueden ser agravante a la aceptación y el compromiso con el embarazo. El objetivo del estudio fue identificar las razones por las que las adolescentes embarazadas que se quedan en las clínicas prenatales. Se utiliza como un enfoque metodológico cualitativo, el enfoque cuantitativo y exploratorio, la muestra estuvo conformada por 15 adolescentes, entre ellos, 10 mujeres embarazadas y 05 posparto. Los resultados podrían inferirse que la mayoría de las mujeres viven en un contexto social de la dependencia financiera completa y que muchos sufren la influencia directa de la familia en la toma de decisiones. Consideramos que el bebé deseos y atención de la salud contribuyó los mayores resultados del índice que motivan a los adolescentes a asistir prenatal.
\end{abstract}

Palabras clave: El embarazo adolescente; Prenatal; Enfermería. 


\section{INTRODUÇÃO}

A gravidez é uma fase singular na vida da mulher que provoca inúmeras transformações hormonais, físicas e emocionais. Seu corpo se modifica e seus hormônios se alteram para a manutenção do feto. Os sentimentos de fragilidade, insegurança e ansiedade normalmente sobrevêm nesta nova etapa de transição feminina. Compreende-se então os temores em relação às alterações na autoimagem corporal, não ter uma criança saudável e a desconhecida função de gerar, nutrir e parir. ${ }^{(1)}$

As mudanças no papel social, na necessidade de novas adaptações, reajustamentos intrapessoais e mudanças de identidade fazem parte da realidade de toda gestante. Quando esse evento ocorre na adolescência essas características rompem simultaneamente com as profundas mudanças comuns da adolescência, caracterizadas principalmente por crescimento rápido, surgimento das características sexuais secundárias, conscientização da sexualidade, estruturação da personalidade, adaptação ambiental e integração social. ${ }^{(2)}$

A adolescência corresponde à faixa etária dos 10 aos 19 anos, ciclo da vida em que ocorre a transição de um estado de dependência para outro de relativa independência. Estes jovens estão em uma condição peculiar de desenvolvimento, pois estão em processo de amadurecimento biopsicossocial. São passíveis também a diversos fatores que predispõem à maternidade extemporânea como: baixa autoestima, dificuldade escolar, abuso de álcool e drogas, comunicação familiar escassa, conflitos familiares, pai ausente e/ou rejeitador, violência física, psicológica e sexual, rejeição familiar pela atividade sexual e gravidez fora do casamento. ${ }^{(1,2)}$

Dados sobre a gravidez na adolescência demonstram um aumento na taxa de fecundidade para esta população quando comparada às mulheres adultas, especialmente nos países mais pobres, como é o caso da América Latina. A gravidez neste grupo populacional pode acarretar complicações obstétricas com repercussões para a mãe e o recém-nascido, bem como problemas psicossociais e econômicos, e vem sendo considerada em alguns países como problema de saúde pública. ${ }^{(2,3)}$

No Brasil a ocorrência da gestação tem uma expressão significativa na idade da adolescência, situação evidenciada pelo Censo 2000 , onde 20,33\% das mulheres gestantes nos 12 meses anteriores estavam na faixa etária entre 10 e 19 anos. Dados de 2007 apontam que aproximadamente $71 \%$ dos nascidos vivos eram filhos de mulheres entre $15 \mathrm{e}$ 29 anos, e os filhos nascidos vivos das mulheres de 15 a 19 anos respondiam por cerca de $20 \%$ do total de nascimentos. ${ }^{(4,5)}$

Os índices atualizados do Sistema Único de Saúde (SUS) revelam que as internações por gravidez, parto e puerpério correspondem a $37 \%$ das internações entre mulheres de 10 a 19 anos. Associado a isso, há o declínio dos índices de gravidez em todas as faixas etárias, exceto entre mulheres de 15 a 19 anos. ${ }^{(6)}$

Segundo dados do DATASUS(7) o número de óbitos maternos entre os 10 e 19 anos de idade foi de 207 casos em 2011 em todo o Brasil. Os casos de mortalidade materna em todas as idades no estado da Bahia de janeiro a junho de 2012 chegaram a 41 óbitos. Esta relação se dá a partir de inúmeras complicações que podem sobrevir às adolescentes no decorrer da gestação e no puerpério como: doença hipertensiva específica da gravidez, infecção urinária, lesões no canal de parto e hemorragias, endometrite, infecções, deiscência de incisões, entre outros. Vale destacar que os casos de complicações do abortamento ilegal comprometem a saúde da mulher e são a causa de 10\% a 15\% de óbitos maternos no Brasil. ${ }^{(2,8)}$

Os eventos adversos gerados pelas complicações durante a gestação podem ser superados e a gravidez bem tolerada, desde que se tenha um acompanhamento pré-natal precoce e adequado durante todo período gestacional. A equipe multiprofissional deve proporcionar um atendimento sistematizado, não oferecendo a assistência à gestante apenas nas dimensões biológicas e curativas, devem ser levados 
em consideração seus sentimentos acerca da experiência em suas múltiplas dimensões. $(3,9,10)$

O pré-natal foi instituído como um programa voltado para a saúde materno-fetal, com intuito de afastar as complicações e diminuir a mortalidade materna e infantil. Dentro da equipe de saúde da família, a (o) enfermeira (o) é um das (os) principais responsáveis pelo acolhimento e pelo andamento das consultas de pré-natal. A enfermagem tem a incumbência de se dedicar a escutar a gestante e transmitir apoio e confiança para que ela persista no acompanhamento e possa conduzir melhor sua gestação. ${ }^{(1)}$

A saúde da adolescente envolve um conjunto de conhecimentos, atividades interdisciplinares e a atuação multiprofissional. Entendemos que para trabalhar com a adolescente, as enfermeiras precisam ser aperfeiçoadas em seus conhecimentos, apresentando uma postura de ética, de modo que as adolescentes possam sentir-se à vontade para expor seus sentimentos, suas dúvidas, angústias e incertezas, destituídas de julgamento de valor moral por parte das profissionais. ${ }^{(3)}$

As experiências passadas com cuidado de saúde, a acessibilidade e aceitabilidade dos serviços identificam fatores que podem intervir positivamente ou negativamente na motivação para manter o cuidado pré-natal regular. São pertinentes que sejam elaboradas estratégias que cativem e mantenham as jovens grávidas no programa e que sirvam de instrumento para captar outras adolescentes não gestantes com a intenção de prevenir a gravidez nesta faixa etária. ${ }^{(3)}$

O que conduz as pesquisadoras a realizarem o trabalho está relacionado com as justificativas anteriormente apresentadas, sendo que as adolescentes grávidas são um público crescente na população atual das maternidades. Além de que a gravidez na adolescência é considerada de risco e passível a complicações para mãe e o feto. Motivos esses que tornam compreensível que o acompanhamento pré-natal é indispensável principalmente para este público.
A pesquisa em questão almeja compreender as razões que conduzem as adolescentes grávidas a permanecerem no atendimento de pré-natal, com o propósito de prevenir os agravos e complicações para o binômio mãe-filho. Demonstrando a importância na identificação e implantação de estratégias que favoreçam o acolhimento e a permanência desse público alvo.

\section{MÉTODOS}

O estudo foi realizado na cidade de Salvador, estado da Bahia, na Maternidade Pública Tsylla Balbino. Construído a partir de um estudo exploratório e descritivo, com abordagem qualitativa pertinente as adolescentes grávidas objetivando interpretar os fenômenos e as causas que mantêm essa população perseverante na assistência pré-natal.

A pesquisa obedeceu a Resolução $n^{\circ} 196$ de 10 de outubro de 1996 do Conselho Nacional de Saúde (CNS) que norteia as pesquisas com seres humanos e foi aprovada pelo Comitê de Ética em Pesquisa da Maternidade Climério de Oliveira.

A linha metodológica foi baseada na etnografia que segundo Godoy, ${ }^{(12)}$ explora um determinado grupo detalhando os eventos relacionados à sua vida envolvendo as estruturas e o seu contexto social.

Os dados analisados neste artigo foram coletados no período de abril a maio de 2013 , todos os dias da semana em que era prestada a assistência pré-natal na unidade (ambulatório de ginecologia e obstetrícia), na roda de conversa de aconselhamento para adolescentes gestantes (no auditório da Instituição) e na assistência prestada as adolescentes puérperas (nas enfermarias). A observação participante foi o método de coleta utilizado, como instrumento usamos um questionário semi-estruturado contendo questões do tipo objetiva e subjetiva. O formulário foi constituído por perguntas que nortearam a pesquisa através dos aspectos pessoais do sujeito e questões subjetivas sobre o tema proposto. O formulário foi aplicado aos sujeitos, constituindo-se das seguintes questões: "Fale 
sobre os motivos que te levaram a assistência de pré-natal", "Houve algum estímulo para que você persistisse na frequência às consultas?", "Quais as suas expectativas para as próximas consultas?". Nestes questionários escolhemos nomear os sujeitos através de nomes fictícios de flores (Jasmim, Flor-De-Lis, Orquídea, Lírio, Rosa Branca, Violeta, Rosa Vermelha, Crisântemo, Margarida, Cravo, Bromélia, Angélica, Espirradeira, Sorriso e Graxa).

Os sujeitos do estudo foram adolescentes grávidas que estavam frequentando o pré-natal na maternidade, puérperas internadas que tenham realizado ou não o pré-natal na maternidade e puérperas que estivessem retornando para a consulta de revisão puerperal. Além desses critérios de inclusão, foi estabelecido que a adolescente deveria participar voluntariamente da pesquisa, assinando o Termo de Consentimento Livre e Esclarecido, estivesse na faixa etária de 10 a 19 anos de idade e que tenha tido uma frequência assídua às consultas pré-natal. O critério de exclusão adotado foi para mulheres a partir dos 20 anos de idade, puérperas adolescentes que frequentaram menos que 04 consultas pré-natais.

É preconizado pelo Ministério da Saúde que sejam realizadas seis consultas ou mais para que o pré-natal seja considerado adequado. A capacitação dos profissionais de saúde para a captação precoce das gestantes para as consultas de pré-natal indica as melhorias na atenção pré e peri-natal. Isso incentiva o cumprimento do recomendado, que se inicie o pré-natal assim que a gestação seja identificada, fortalecendo a adesão da mulher ao pré-natal e promovendo o diagnóstico precoce de eventuais fatores de riscos. ${ }^{(13)}$

A amostra do grupo de estudo foi formada por dois grupos participantes: As gestantes que fazem o acompanhamento pré-natal na Instituição e as adolescentes puérperas internadas que fizeram ou não acompanhamento pré-natal na Maternidade, sendo que durante o período da pesquisa não houve puérperas adolescentes para o retorno de revisão puerperal.
Acompanhamos inicialmente as adolescentes durante as rodas de conversa e nas consultas pré-natal almejando estabelecer um contato mais próximo para favorecer a abordagem dessas no momento da coleta. As rodas de conversa é uma estratégia utilizada na Maternidade que favorece $o$ acolhimento das jovens adolescentes e seus familiares, tornando um vínculo de confiança, esclarecendo suas dúvidas, medos e anseios. Após a etapa anterior, conhecemos as unidades de internação delimitando em quais enfermarias seria viável a coleta de dados das adolescentes puérperas. Posteriormente, em primeira instancia foram abordadas as puérperas e ao concluir esta etapa continuamos as coletas com as gestantes no ambulatório que foram acompanhadas desde a chegada à recepção até o término da consulta pré-natal e neste momento a abordamos e aplicamos os questionários.

No período da coleta as consultas eram realizadas por uma médica que as assiste por serem grávidas na classificação de risco.

\section{DESENVOLVENDO A ANÁLISE DOS DADOS}

Utilizado para a análise do conteúdo a vertente temática que é subdividida em três etapas (Pré-análise, exploração dos materiais e o tratamento dos resultados. (14) $\mathrm{Na}$ "pré-análise" foi feita a organização dos materiais coletados, separando e marcando os mais relevantes do estudo. A "exploração dos materiais" serviu para categorizar as informações, encontramos quatro categorias (Perspectivas sobre a saúde e desenvolvimento fetal, $A$ influência da família durante o acompanhamento pré-natal das adolescentes, Curiosidade sobre a saúde gestacional, Assistência humanizada prestada pelos profissionais). O "tratamento dos resultados" revelou-os a partir da condensação de dados destacando as informações para posterior análise critica e reflexiva.

O número de gestantes que compuseram o estudo foi de 15 adolescentes, dentre elas 10 foram gestantes e 5 puérperas. Abrangemos na pesquisa 
gestantes na faixa etária dos 14 aos 19 anos. Prevaleceram neste público adolescentes com 15 anos apresentando um percentual de $34 \%$ (05), enquanto $7 \%$ (O1) tinham 14 anos compondo a menor faixa etária do grupo.

Referente a classe racial, $67 \%$ (10) se autodeclararam negras, $27 \%$ (04) pardas e a menos declarada com 6\% (O1) branca. Ao falar sobre a escolaridade a maioria das adolescentes referiam o abandono escolar no ensino fundamental ou $1^{\circ}$ grau após inicio da gestação.

Muitas adolescentes declaravam serem solteiras, porém ao ser explicado o questionamento identificamos que estas viviam em união consensual $(67 \%)$, dentre essas $47 \%$ moram com o companheiro e $33 \%$ moram com o companheiro e outros familiares, as solteiras compunham $20 \%$ e todas elas moram com familiares, sendo apenas $13 \%$ as casadas.

Apesar do número crescente de multíparas na adolescência constatamos que nesta amostra 93\% (14) eram primigestas e apenas 7\% (1) já tinha um filho. Um fato que nos marcou no momento das entrevistas é que muitas referiam da seguinte forma: "Esse é primeiro e último filho!", isso nos fez perceber que possivelmente elas não tiveram uma gestação planejada ou viveram este momento de forma pouco prazerosa.

Durante o momento em que passamos com essas adolescentes percebemos que ao falarmos sobre a remuneração a maioria delas não expressava maturidade suficiente respondendo de forma insignificante sobre o seu papel de provedora, no planejamento financeiro, durante a gravidez para seu filho. Para demonstrar, em média $80 \%$ delas não vivem às próprias custas, $20 \%$ são independentes financeiramente, sendo que $13 \%$ trabalham fora de casa e apenas 01 vive às custas de pensão.

\section{PERSPECTIVAS SOBRE A SAÚDE E DESENVOLVIMENTO FETAL}

Sobre a importância do pré-natal no período gravídico interpretamos que as falas e expressões das adolescentes remetiam uma preocupação exclusiva com o bebê, entretanto, evidenciando uma imaturidade e inexperiência acerca da importância da saúde gestacional em relação ao desenvolvimento fetal.

"[...] A saúde do meu bebê em primeiro lugar, e... só! Em primeiro lugar é o bebê né? (risos)". (Flor Margarida)

"Para proteger o bebê saber como ele tá, só isso." (Flor Graxa).

"[...] Foi pra que meu bebê nasça com saúde [...]." (Flor Sorriso).

Segundo Moreira( ${ }^{(1)}$ compreende-se então que os temores das adolescentes grávidas em relação às alterações na autoimagem corporal não terem uma criança saudável e a desconhecida função de gerar, nutrir e parir.

Através dos risos e pausa no momento das respostas foi possível observar a timidez em expressar o real sentimento sobre o que foi argumentado e/ou incerteza do que responder. Outra evidência disso está em uma das respostas, na qual a adolescente somente respondeu a arguição após o estímulo de um parente que estava presente no momento da entrevista.

"(Risos)... Ai meu Deus! Por causa do meu fiIho... a saúde dele." (Flor Rosa Vermelha).

"É (pausa)... Saber como tá meu filho, como estão as coisas, se ele tá bem. (Flor Jasmim).

[...] Ahh!! Saúde do nenê. (Flor Rosa Branca).

[...] "Pá vê" como é que tá as coisas, o desenvolvimento. (Flor Bromélia).

O período gravídico é naturalmente constituído por susceptibilidades para a saúde da mãe, bem como a do concepto. A concepção de um filho na adolescência intensifica a probabilidade de eventos de riscos biológicos e psicológicos para a saúde de ambos, devido às mudanças peculiares que ocorrem nessa fase de transição como as mudanças físicas, 
hormonais e emocionais características da adolescência. ${ }^{(15)}$

\section{A INFLUÊNCIA DA FAMÍLIA DURANTE O ACOMPANHAMENTO PRÉ-NATAL DAS ADOLESCENTES}

Em muitos depoimentos observamos que a família expressou um papel de influência sobre a decisão das adolescentes e em alguns casos de imposição para que essas frequentassem assiduamente 0 pré-natal.

"É... Minha mãe me incentivando sempre, dizendo que é melhor para mim e para o bebe." (Flor-De-Lis).

"Porque a amiga de minha mãe diz que é muito boa e também por causa de minha avó. Porque disse que se eu não tivesse feito o Pré-natal poderia ocorrer alguma coisa assim na hora, na hora que eu for ter a criança." (Flor Lírio).

"Minha sogra que mandou procurar". (Flor violeta).

"Minha mãe primeiro que fala que só, aí fica falando que precisa pra saber, do bem estar do bebe, se ele está precisando de alguma coisa e a família que fica falando toda hora". (Flor Espirradeira).

O enfrentamento do complexo período vivenciado pela adolescente grávida no aspecto psicológico revela grande vulnerabilidade aos transtornos mentais, sendo a depressão o transtorno mais trivial entre as mulheres grávidas e que, no entanto acomete mais as adolescentes do que as adultas. Secundário a esse sofrimento psicológico os agravos que podem prejudicar a saúde e desenvolvimento do bebê estão a prematuridade, o baixo peso ao nascer e retardo no desenvolvimento da criança. Os fatores que influenciam no desencadeamento de algumas dessas complicações, que podem ser consideradas, sócio biológicas, podem estar relacionados em alguns casos ao nível de escolaridade, estado civil, apoio familiar e, sobretudo, com um inadequado acompanhamento de pré-natal. A falta de suporte físico e apoio psicológico no processo gestacional podem levar a adolescente a se expor a hábitos de risco para sua saúde e a saúde fetal, como o uso de álcool, drogas e o fumo, doenças sexualmente transmissíveis, anemia, desnutrição, abuso sexual e físico. ${ }^{(11)}$

Conclui-se que as práticas educativas parentais inferem valiosamente no desenvolvimento de atitudes, valores, comportamentos e sexualidade dos adolescentes. ${ }^{(16)}$

\section{CURIOSIDADE SOBRE A SAÚDE GESTACIONAL}

Poucas adolescentes preocuparam-se com a própria saúde, enquanto gestantes. Dentre todas, duas deram a subentender que sua preocupação era secundária a um problema de saúde pré-existente e à falta de check-up da saúde.

“Eu procurei[...]porque eu também tenho problema de anemia. Aí porque podia passar pra nenê também". (Flor Crisântemo)

"[...] para saber se eu estou com saúde. Porque aí também tem muito tempo que eu não faço exames". (Flor Sorriso)

Em sua pesquisa com adolescentes grávidas Ribeiro ${ }^{(17)}$ percebeu que o comportamento deste público demonstra claramente a necessidade de aquisição de mais informações no que concerne à sua assistência pré-natal.

Estudos revelam que o acompanhamento das adolescentes gestantes nas consultas de pré-natal inicia de forma tardia e que na maioria das vezes é frequentado em um número inferior ao preconizado. O histórico social, a falta de apoio familiar, a baixa escolaridade são alguns dos fatores que contribuem para a escassez na atenção desse grupo etário. ${ }^{(18)}$ 


\section{ASSISTÊNCIA HUMANIZADA PRESTADA PELOS PROFISSIONAIS}

As percepções das usuárias quanto ao serviço de pré-natal representam um elemento determinante para a valorização deste serviço. Sendo, portanto, a humanização considerada como princípio da qualidade da assistência, foi possível entender que a falta de atendimento no final da gestação, ou seja, a procura de uma "alta do pré-natal", é um possível agente causador da desistência do curso das gestas adolescentes ao pré-natal. ${ }^{(19,20)}$

"A médica me estimula a todo mês está aqui fazendo o pré-natal". (Flor Orquídea)

"A doutora que é muito boa e minha avó que ficou me estimulando". (Flor Lírio)

"Gostei!...porque eu gostei do atendimento" (Flor Violeta)

A forma de abordagem da equipe através das rodas de conversa pretende promover informações e um vínculo de credibilidade com este público. Essa estratégia serve para otimizar o retorno dessas adolescentes às consultas de rotina pré-natal, tornando a consulta um momento agradável e estimulante.

A jornada das mulheres a procura de maternidades para parir, ressalta a deficiência na questão da falta de vínculo entre o pré-natal e a assistência ao parto. Lacuna esta que está sendo atualmente preenchida pela inovadora estratégia do Ministério da Saúde conhecida como Rede Cegonha, lançada no Brasil no ano de 2011. Através da ampliação do acesso e da melhoria da qualidade do pré-natal, da vinculação da gestante à unidade de referência e ao transporte seguro, da implementação de boas práticas na atenção ao parto e nascimento, incluindo o direito ao acompanhante de livre escolha da muIher no parto, da atenção à saúde das crianças de o a 24 meses e do acesso às ações de planejamento reprodutivo. ${ }^{(21)}$

A assistência obstétrica à mulher passou por diversas adequações para minorar as carências en- contradas no serviço prestado pela rede pública de saúde. Aprovisionar a qualidade da assistência, o acesso aos serviços fornecidos e a humanização da atenção no pré-natal e ao parto, constituíram o diferencial ao longo dos processos de melhoria nesse conjunto de ações básicas oferecido pelos municípios. ${ }^{(13)}$

A adolescente ao procurar a unidade de saúde, não é diferente de qualquer outro sujeito. $O$ indivíduo está em busca da escuta e atendimento das suas necessidades, demonstrando sentir confiança e amparo pela equipe de saúde. Enquanto é possível também encontrar, principalmente na faixa etária da adolescência e entre adolescentes grávidas, dificuldade em procurar a unidade de saúde por se sentir intimidado, envergonhado ou temeroso de ser repreendido pelo profissional. ${ }^{(22)}$

\section{CONSIDERAÇÕES FINAIS}

Para encontrarmos os resultados apresentados foi necessário analisarmos secundariamente aspectos obscuros que não puderam ser compreendidos apenas através das falas dos sujeitos, mas através de suas expressões faciais e entonações vocálicas.

Ficou esclarecido que mediante todos os fatores que interferem sob as motivações das adolescentes quanto ao pré-natal, no geral elas são vulneráveis não possuindo iniciativa própria na persistência às consultas. Mesmo sendo empírico para elas o que se refere à saúde do bebê, entendem que é um fator positivo continuar o esquema pré-natal, para que, como referido por algumas delas nada de ruim aconteça com a criança.

A imposição familiar repercutiu nessas adolescentes como um "megafone" chamando a atenção para a importância de acompanhar a saúde fetal e ser preparada para o momento do parto. Em concordância com este fato, o acolhimento adequado dos profissionais envolvidos sobressaiu como fator estimulante para que essas jovens retornassem às consultas e ao acompanhamento multiprofissional até o final da gestação. 
Por fim, a vivência com essas adolescentes nos mostrou a carência existente em relação à educação e planejamento familiar, muitas delas vivem o que na maioria das vezes suas mães viverem. É importante que essas jovens estejam mais preparadas para esse momento único de suas vidas, que muitas vezes não é indesejado, mas inoportuno e que os profissionais envolvidos neste processo possam acolher essas jovens de maneira adequada sem julgamentos ou criticas.

As motivações foram identificadas e vale à pena que estas sejam alimentadas, aumentando as estratégias de acolhimento dos profissionais, envolvendo diretamente a família que simboliza um forte papel nesta fase da vida delas. Assim almejamos, que se aplicadas estratégias próprias para este público, o índice de adesão ao pré-natal poderá ser muito mais satisfatório, causando um benefício direto na prevenção das complicações gestacionais na adolescência.

\section{REFERÊNCIAS}

Moreira TMM, Viana DS, Queiroz MVO, Jorge MSB. Conflitos vivenciados pelas adolescentes com a descoberta da gravidez. Rev. Esc. Enferm. USP. 2008;42(2):312-2O.

Yazlle MEHD. Gravidez na adolescência. Rev. bras. ginecol. obstet. 2006;28(8):443-45.

Clapis MJ, Parenti PW. O conhecimento produzido acerca da assistência à adolescente grávida. Rev. bras. enferm. 2004;57(3):284-87.

Esteves JR, Menandro PRM. Trajetórias de vida: repercussões da maternidade adolescente na biografia de mulheres que viveram tal experiência. Estud. psicol. 2005;10(3):363-70.

IPEA. Juventude e políticas sociais no Brasil. Brasília: Ipea; 2009.

Brasil. Ministério da Saúde. Secretária de Atenção a Saúde. Marco teórico e referencial: saúde sexual e saúde reprodutiva de adolescentes e jovens. Brasília, DF: Ministério da Saúde; 2006.

DATASUS - Departamento de Informática do SUS [Internet]. Brasília: Ministério da Saúde; [acesso em
2006 out. 20]. Disponível em: http://svs.aids.gov.br/ dashboard/mortalidade/materna.show.mtw

Chaves JHB, Pessini L, Bezerra AFS, Rego

G, Nunes R. A interrupção da gravidez na adolescência: aspectos epidemiológicos numa maternidade pública no nordeste do Brasil. Saúde Soc. 2012;21(1):246-56.

Duarte SJH, Andrade SMO. Assistência pré-natal no Programa Saúde da Família. Esc. Anna Nery Rev. Enferm. 2006;10(1):121-25.

Brasil. Ministério da Saúde. Secretaria de Atenção à Saúde. Pré-natal e puerpério: atenção qualificada e humanizada - manual técnico. Brasília, DF: Ministério da Saúde; 2005.

Pereira PK, Lovisi GM, Lima LA, Legay LF. Complicações obstétricas, eventos estressantes, violência e depressão durante a gravidez em adolescentes atendidas em unidade básica de saúde. Rev. psiquiatr. clín. 2010;37(5):216-22.

Godoy AS. Pesquisa qualitativa: tipos fundamentais. Revista. adm. empres. 1995;35(3): 20-29.

Serruya SJ, Lago SJ, Cecatt JG. O panorama da atenção pré-natal no Brasil e o Programa de Humanização do Pré-natal e Nascimento. Rev. bras. saúde matern. infant.2004;4(3):269-79

Grzybovski D, Mozzato AR. Análise de conteúdo como técnica de análise de dados qualitativos no campo da administração: potencial e desafios. RAC. 2011;15(4):731-47.

Moraes ML, Almeida LB, Santo RE, Barbosa RF, Carmo MGT. Elementos traço e complicações obstétricas na gestação na adolescência. Rev. nutr. 2010;23(4):621-28.

Ceconello AM, Antoni C, Koller SH. Práticas educativas, estilos parentais e abuso físico no contexto familiar. Psicol. estud. Maringá. 2003;8:4554.

Ribeiro RS et al. A visão das adolescentes gestantes sobre o pré-natal: qualificação da atenção e dos recursos humanos de enfermagem em saúde da mulher e do recém-nascido In: Anais do VI Congresso Brasileiro de Enfermagem Obstétrica e Neonatal, 2009. Teresina: ABENFO, 2009.

Gama SGN, Szwarcwald CL, Sabroza AR, Branco VC, Leal MC. Fatores associados à assistência pré-natal precária em uma amostra de puérperas adolescentes em maternidades do Município do 
Rio de Janeiro. 1999-2000. Cad. saúde pública. 2004;20(1):101-11.

Brasil. Ministério da Saúde. Secretaria de Políticas de Saúde. O desafio de construir e implementar políticas de saúde: relatório de gestão 2000-2002. Brasília, DF: Ministério da Saúde; 2002. 220 p. Leão EM, Marinho LFB. Saúde das mulheres no Brasil: subsídios para as políticas públicas de saúde. Rev. Promoção da Saúde. 2002; (3): 31-36.
Brasil. Ministério da Saúde. Secretaria da Saúde. Departamento de Ações Programáticas Estratégicas, Área Técnica de Saúde da Mulher. Rede Cegonha: diretrizes gerais e operacionais da rede cegonha. Brasilia, DF: Secretaria de Saúde, 2011.

Brasil. Ministério da Saúde. Secretaria da Saúde. Coordenação de Desenvolvimento de Programas e Políticas de Saúde-CODDEPS. Manual de atenção à saúde do adolescente. Brasília, DF: Secretária Municipal de Saúde de São Paulo, 2006. 328 p. 